\title{
Study on Characteristics of Pedestrian Crossing the Street in Shijingshan
}

\author{
Weiwei Guo ${ }^{1, \mathrm{a}}$, Xin Ma ${ }^{1, \mathrm{~b}}$, Yinghong $\mathrm{Li}^{1, \mathrm{c}}$, Jiyuan $\operatorname{Tan}^{1, \mathrm{~d}}$,Yan Zhang ${ }^{1, \mathrm{e}}$ \\ ${ }^{1}$ Beijing Key Lab of Urban Intelligent Traffic Control Technology, North China University of \\ Technology, Beijing, 10014, China

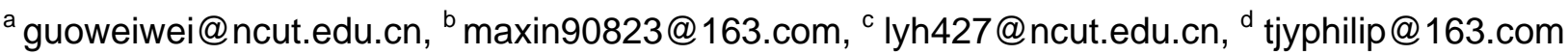

Keywords: Pedestrian, Cross the street, Violation, Maximum red time, Survival analysis

Abstract. The problem of pedestrian across the street during the red light is always a focus of China's urban traffic management and difficult. This phenomenon has been improved after years of renovation in some developed cities such as Beijing. However, in some middle-sized and small cities, the problem of pedestrian across the street during the red light is still serious. Pedestrians as vulnerable groups of the traffic participants, more likely hurt by motor vehicle. The problem of pedestrian across the street during the red light is not only a violation of traffic regulations and seriously affect traffic order, increasing the possibility of accidents at the same time. Pedestrian traffic is the weaknesses of urban traffic system, it's meaningful to study on characteristics of pedestrian crossing. We used survival analysis for modeling the process of pedestrian's waiting. According to the survey data of the waiting time which the pedestrians across the street in Shijingshan, we acquire a survival curve of waiting time in three groups of old people, middle-aged people and young people. The result shows that the pedestrians on the roadside waiting to cross the street is 34s, and recommend that the maximum red time in Shijingshan is less than 50s. Reasonable control can improve compliance rates, and reducing the risk of violation.

\section{Introduction}

Pedestrian traffic as an important part of urban transportation is more and more taken into account by urban managers. Country and local governments are in support of "people-oriented" traffic design, Due to the imbalance of economic development, large number of population, even the economic development rapidly, still can't change the traffic pattern which urban residents' mainly ways to travel is walking and cycling, this situation could not be changed radically in a short term ${ }^{[1]}$.

Pedestrian traffic as the city of "vulnerable groups" is vulnerable to some factors. In the Act of crossing the street, male and women have obviously different behaviors. Emotions effect the proportion which pedestrians arise dangerous behavior, happy mood will improve traffic security behavior ${ }^{[2]}$. The factors of age also effect with characteristics when pedestrian crossing the street. Elderly due to physiological reasons, waiting time is more long, and can't take enough attentions of traffic environment ${ }^{[3,4]}$. In today social, telephone has widely used, however, using telephone when crossing the street makes walking slowly, which ignore the traffic status around us, and cognitive capacity declined ${ }^{[5]}$.

At the studies of violation and the waiting time of pedestrian crossing the street, United Kingdom N. Rouphailn, who respectively to signal control and research was carried out at no signal control intersections, including 164 cases pedestrian crossing delays and forced through the survey, found that men were more likely than women in violation, locals' unbearable limits waiting time is 45 60s [6]. India Geetam Tiwari, successful application of survival analysis, selecting some typical intersections of pedestrians crossing the street in New Delhi waiting time and risk-taking to study, when the waiting time exceed the limit, it may be illegal across the street ${ }^{[7]}$.

Therefore, in order to improve pedestrian safety and diminish the violation ratio of regulation, the paper analyses the regularity of pedestrian waiting behavior and the study of pedestrian waiting time under signal control and the critical gap was estimated using the refused and accepted gap data of pedestrian crossing collected. 


\section{Kaplan-Meier Estimation Method}

Survival analysis is on the basis of comprehensive consideration of relevant factors(internal and external causes).Related to biology, medicine, engineering, actuarial science, public health, sociology, demography, economics and other fields, and problems related to the time of the incident related to the statistical laws analysis and inference methods of discipline ${ }^{[8]}$.

During the red light, pedestrians reach and pass to study the interest of events, study on the regular of the survival of their waiting time. Research object is the cross-street pedestrian. "Death " for pedestrians crossing the street open to traffic. Pedestrians wait time is defined as pedestrians to reach the crossing waiting area began passing time.

Arrived at the intersection of different signal color, pedestrian crossing the street can be divided to the following 3 categories:

The first category, reached during the green light. The pedestrian immediately cross.

The second category, green flash period arrives, which pedestrians have 3 options.

(1) Immediately pass.

(2) Wait for the next cycle of green time to pass.

(3) Pedestrians in the process of waiting for the next cycle, due to lost patience, and pass the crossing even the red light had reached.

The third category, pedestrians arrive when the red light reaches. Such pedestrian has two choices:

(1) Wait for the next cycle of green time to pass.

(2) After waiting for some time at a red light to pass.

When the pedestrians arriving or waiting for a period of time and choice to pass during the red light.

The first pedestrian have no signal delay, through observe the waiting regular of the second pedestrian, A total of 257 people in observing the videos arrive when the Green light, a total of 252 people select immediately to pass ,it's nearly 98.6\%, The pedestrian select who waiting for the next cycle of green time to pass and to pass during the red light are 1 person and 4 person, nearly $98.6 \%$ the second pedestrian's waiting time are 0 . Therefore, The study does not take into account the regular of survival of waiting time in the second pedestrian, and then choose the three pedestrian as research object $^{[6]}$.

According to the differences of observed time which observed object into the observation or exit the observation. Survival analysis frequently encounter data is completely data and censored data. Complete data refers to the pedestrian violations during the observed period(end event), then we have the complete time information, this survival time data is called complete data; Censored data refers to the pedestrians during the waiting period, after the light turns green to get normal pass without the violation phenomena were observed. Now recorded temporal information is incomplete, the survival time of data called censored data.

Samples in this study of the waiting time which pedestrians cross the street convey a lot of censored data. If waiting time is normally, we use $X i$ to express; if waiting time is censored data, then we use $C_{r i}$ to express it. Obviously, censored data includes the tolerable waiting time information of pedestrian crossing the street. Therefore, we need to integrate this data with the complete data to get a modeling.

Survival function reflect that the probability of individual survival time until $\mathrm{x}$ (an experience after an incident at the moment of $\mathrm{x}$ ), the survival function of pedestrian waiting time is that the proportion of pedestrian who choose to continue to wait after waiting for $t$ time.

The survival function is the integral of the probability density function:

$$
S(x)=P(X \geq x)=\int_{x}^{\infty} f(t) d t
$$

Of Which, $\mathrm{P}(\mathrm{X}>\mathrm{x})$ represents the event, and $\mathrm{P}(\mathrm{X}>\mathrm{x})$ represents probability of occurrence.

The graph of $S(x)$ is the survival curves, Sort for each follow-up individuals' time of death, if I-th was truncated data, that is 0 . If I-th was Non - truncated data, after sort the probability of death with no difference, probability of the I-th variable is less than variable t in the observation point ,therefore, 
the estimated on survival function $S(t \mid x)$ of all observations is any variables' multiplication of the survival probability.

\section{Calculation procedure of the Estimation Method}

As for the survival function of pedestrian waiting times when they cross the street which contains the censored data, the steps for calculating are as follows when using Kaplan-Meier Estimation Method.

Step 1: Make observations (Survival Time) of total $\mathrm{N}$ sample from small to large in order of priority, The number of iterations $i=1,2,3 \ldots . .$. When the values of non-censored are equal to the censored, the censored values come first.

Step 2: List the number of pedestrian when observation begin, the number of traffic violation and censored by using of each stage (take 10s as a unit).

Step 3: Calculate death and survival probability of each moment, using

$$
q_{i}=d_{i} / n, p_{i}=1-q_{i}
$$

Step 4: Calculate the rate of survival of pedestrians when

$$
S\left(t_{i}\right)=\prod_{j=1}^{i} p_{j}
$$

Step 5: Draw the curves of survival rate, mark the non-censored value and the survival rate which non-censored value correspond in the Cartesian coordinate system, then make the points vertically down and go horizontally rightwards to make into ladder.

\section{Data processing and analysis}

Grouped respondents according to different ages, taking into account the classification of the popular crowd, objects classified as elderly, middle-aged and young people into three groups. Here we list only the older data, other data for pedestrians is no longer listed.

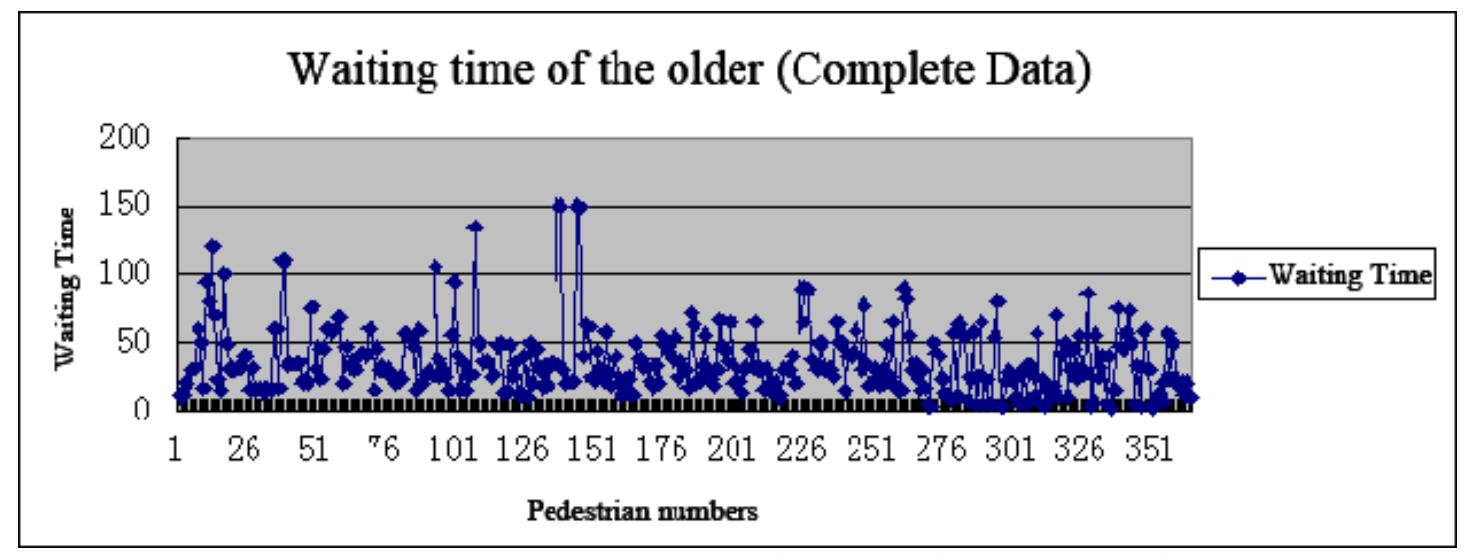

Fig 1.The waiting time of the older (Complete data)

As shown in Fig 1 ,there are 54 old people which only conveys complete data, It isn't too hard to see,the average waiting time is $24.74 \mathrm{~s}$. 


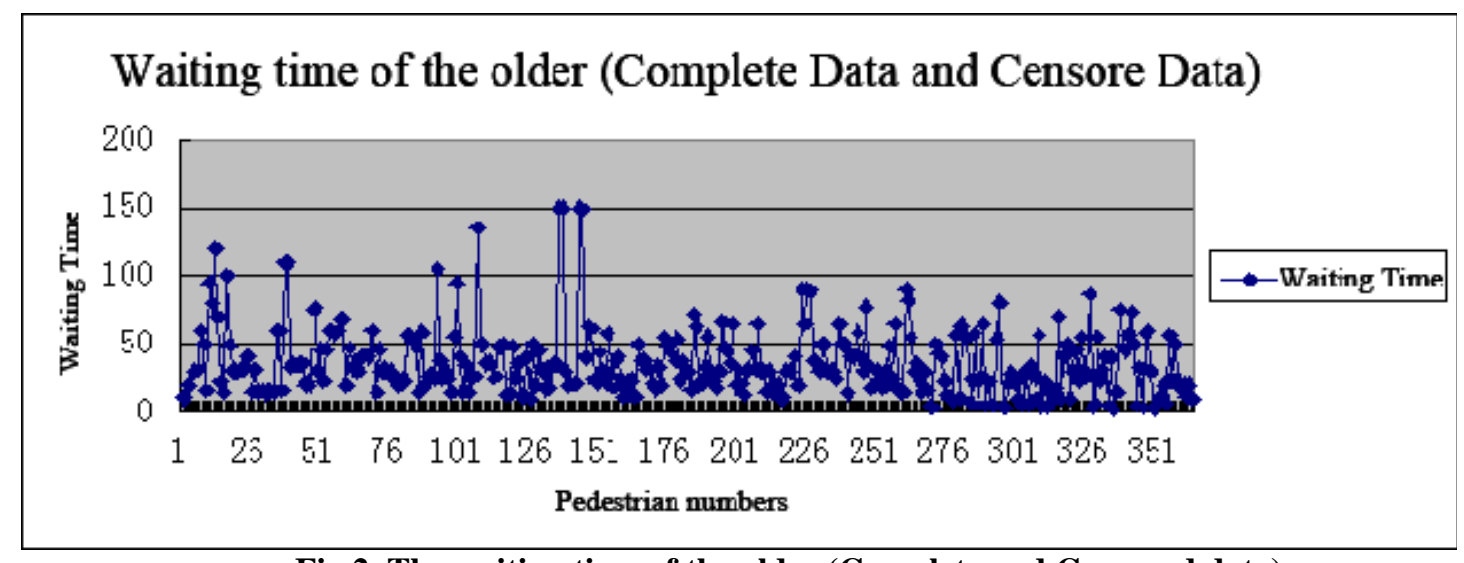

Fig 2. The waiting time of the older (Complete and Censored data)

As shown in Fig 2, there are 78 old people which not only convey complete data, but also have censored data. It isn't too hard to see,the average waiting time is 32.88s.

According to the Kaplan-Meier estimation of survival function to calculate, obtained the data in Table 1.

Table 1.The table of pedestrian survival rate (Consider censored Data)

\begin{tabular}{|c|c|c|c|c|c|c|}
\hline $\begin{array}{l}\text { Wait time } \\
\text { interval }\end{array}$ & $\begin{array}{c}\text { Initial number of } \\
\text { waiting }\end{array}$ & $\begin{array}{l}\text { Number of } \\
\text { censored }\end{array}$ & $\begin{array}{l}\text { Number of } \\
\text { violations }\end{array}$ & $\begin{array}{l}\text { Violation } \\
\text { Probability }\end{array}$ & $\begin{array}{c}\text { Probability of } \\
\text { compliance }\end{array}$ & Live the survival rate \\
\hline$[0,10)$ & 365 & 0 & 26 & 0.071 & 0.929 & 0.929 \\
\hline$[10,20)$ & 339 & 13 & 45 & 0.133 & 0.867 & 0.805 \\
\hline$[20,30)$ & 281 & 27 & 49 & 0.174 & 0.826 & 0.665 \\
\hline$[30,40)$ & 205 & 38 & 39 & 0.19 & 0.81 & 0.539 \\
\hline$[40,50)$ & 128 & 19 & 22 & 0.172 & 0.828 & 0.446 \\
\hline$[50,60)$ & 87 & 20 & 17 & 0.195 & 0.805 & 0.359 \\
\hline$[60,70)$ & 50 & 18 & 4 & 0.08 & 0.92 & 0.33 \\
\hline$[70,80)$ & 28 & 8 & 1 & 0.036 & 0.964 & 0.318 \\
\hline$[80,90)$ & 19 & 4 & 0 & 0 & 1 & 0.318 \\
\hline$[90,100)$ & 15 & 5 & 0 & 0 & 1 & 0.318 \\
\hline$[100,110)$ & 10 & 1 & 1 & 0.1 & 0.9 & 0.287 \\
\hline$[110.120)$ & 8 & 2 & 0 & 0 & 1 & 0.287 \\
\hline$[120,130)$ & 6 & 1 & 0 & 0 & 1 & 0.287 \\
\hline$[130,140)$ & 5 & 1 & 0 & 0 & 1 & 0.287 \\
\hline$[140,150)$ & 4 & 1 & 0 & 0 & 1 & 0.287 \\
\hline$[150,160)$ & 3 & 3 & 0 & 0 & 1 & 0.287 \\
\hline
\end{tabular}

Get the survival curve of pedestrian waiting which concerned censored data in Fig 3. 


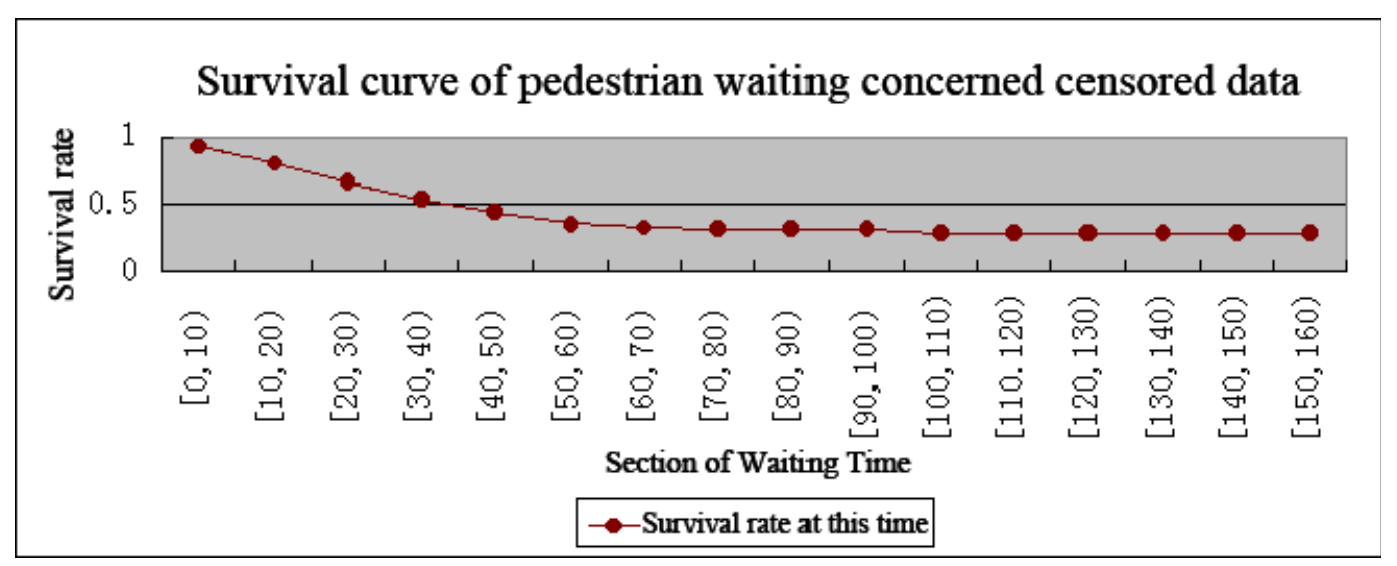

Fig 3.Survival curve of pedestrian waiting concerned censored data

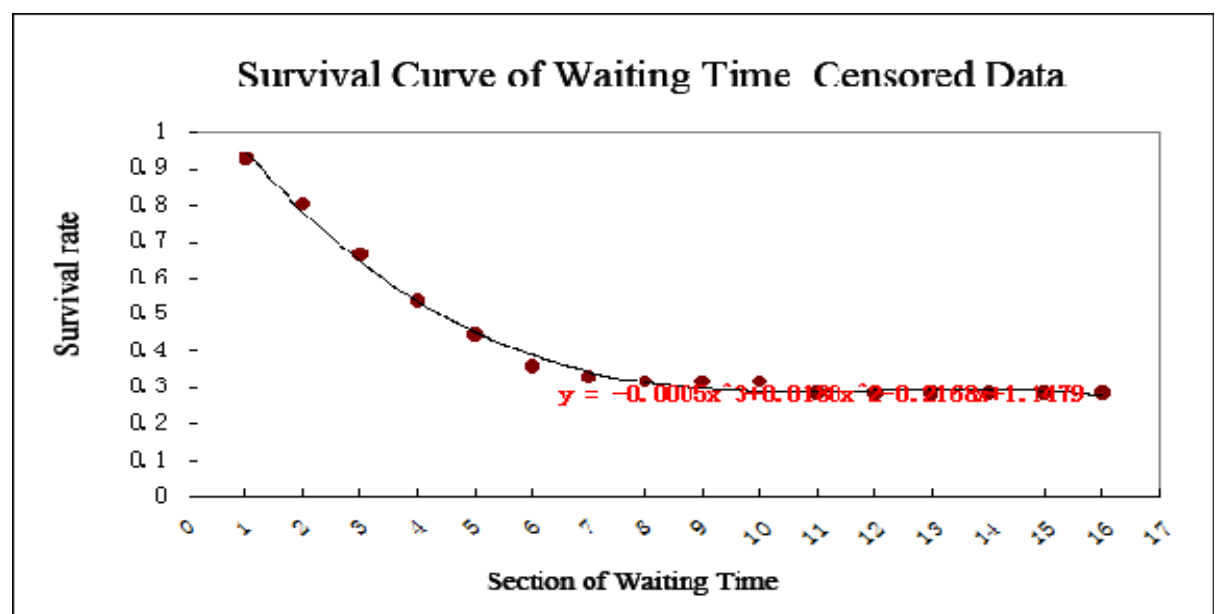

Fig 4.A scatter of chart survival curve

On the base of Fig 3, Set the chart type to a scatter chart, and add a trend line, then we can get Fig 4. Get a regressed polynomial:

$$
y=-0.0005 x 3+0.018 x 2-0.2168 x+1.1479
$$

Using simulation analysis-goal to seek and get the results:

Index: 4.40

The target of the survival rate: 0.5

Waiting time: $34.0 \mathrm{~s}$

According to the survival function of Kaplan-Meier estimation method, making a similar calculate, which the pedestrian survival rate regardless of the censored data.

Table 2.The table of pedestrian survival rate (Not consider censored Data)

\begin{tabular}{|c|c|c|c|c|c}
\hline Wait time interval & $\begin{array}{c}\text { Initial number of } \\
\text { waiting }\end{array}$ & $\begin{array}{c}\text { Number of } \\
\text { censored }\end{array}$ & $\begin{array}{c}\text { Number of } \\
\text { violations }\end{array}$ & $\begin{array}{c}\text { Violation } \\
\text { Probability }\end{array}$ & Probability of compliance \\
\hline$[0,10)$ & 203 & 26 & 0.128 & 0.872 & 0.872 \\
\hline$[10,20)$ & 177 & 45 & 0.254 & 0.746 & 0.65 \\
\hline$[20,30)$ & 132 & 49 & 0.371 & 0.629 & 0.409 \\
\hline$[30,40)$ & 83 & 39 & 0.47 & 0.53 & 0.217 \\
\hline$[40,50)$ & 44 & 22 & 0.5 & 0.5 & 0.108 \\
\hline$[50,60)$ & 22 & 17 & 0.773 & 0.227 & 0.025 \\
\hline$[60,70)$ & 5 & 4 & 0.8 & 0.2 & 0.005 \\
\hline$[70,80)$ & 1 & 1 & 1 & 0 & 0 \\
\hline$[80,90)$ & 0 & 0 & & & \\
\hline
\end{tabular}


Get the survival curve of pedestrian waiting which not concerned censored data in Fig 5.

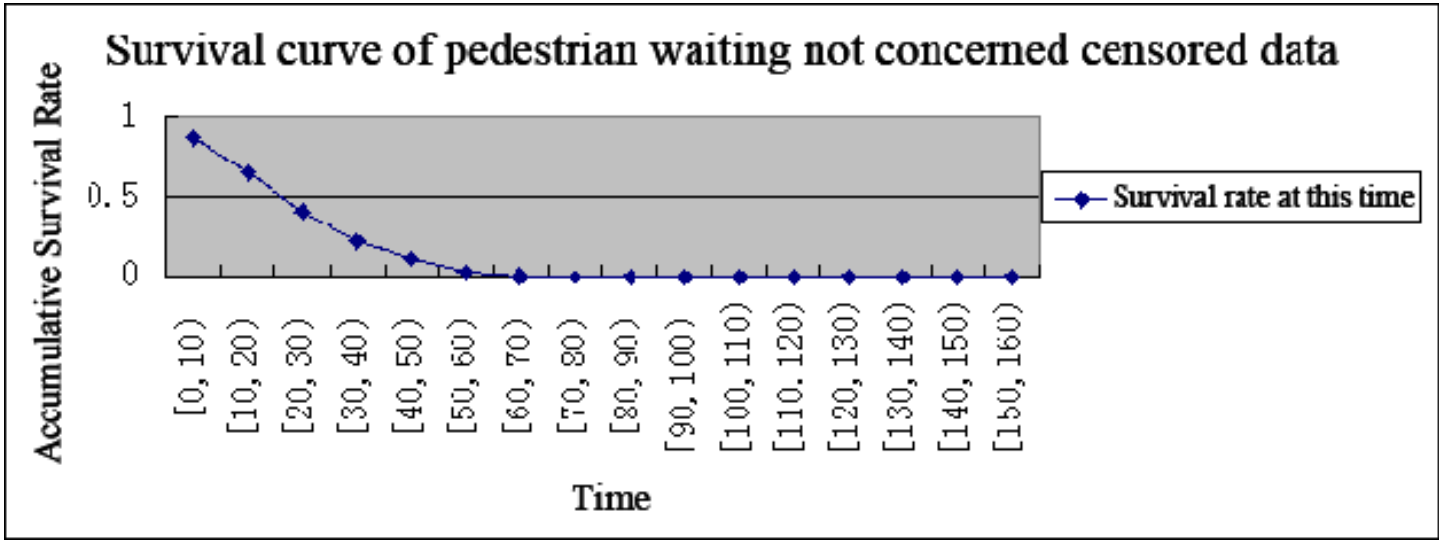

Fig 5. Survival curve of pedestrian waiting not concerned censored data

The same algorithms considered censored data, when regress censored data, the mean value of the survival time which the pedestrians waited.

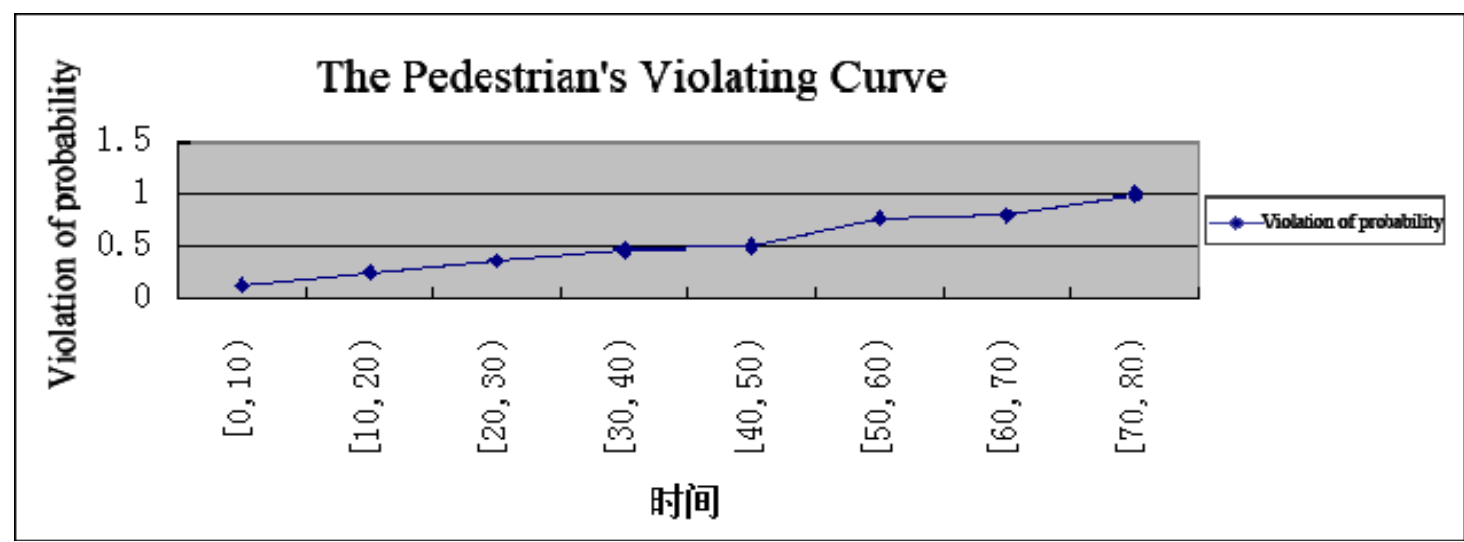

Fig 6. The censored data curve regardless of pedestrian's violating

It's easily to discover from Fig 6, which the longer we wait, the higher violation probability of pedestrian, the average violation rate is 0.537 .

\section{Summary}

According to a large number of video observations, pedestrian arrived in the period of flashing green, 98. 6\% of pedestrian choose to pass immediately. The pedestrians who arrived during the red as the research object, studying the regular of it is violation, and we can analysis the observed sample by using survival analysis.

1. When consider censored data, the average waiting time of pedestrians is $35.9 \mathrm{~s}$, the average of living time is $34.0 \mathrm{~s}$.

2. When not consider censored data, the average waiting time of pedestrians is $26.6 \mathrm{~s}$, the average of living time is $21.0 \mathrm{~s}$.

3. Obviously, the two curves are very different, in other words, if we don't consider the impact of censored data, the average waiting time of pedestrian in violation about $13 \mathrm{~s}$.

4. Observations indicate that higher risk of older people when crossing the street, the lower danger of young people to pass the street. Therefore, we should focus on the older people's safety awareness by the propaganda and education of traffic safety.

5. Reasonable intersection design and signal control can improve compliance rates, reducing the risk of violation. It's recommended that the largest red light time of pedestrian in Shijingshan intersection, which shouldn't more than the 45s. 


\section{Acknowledgements}

This research is partially supported by the National Natural Science Foundation of China (No. 61503007), the Start-up Fund Project of North China University of technology (NCUT2015002), Beijing Talents Training funding (No.2014000020124G008), Education Committee (PXM2015_014212_000023), and Project of Science and Technology of Beijing City (Z141100000714002). The authors gratefully thank anonymous referees for their useful comments and editors for their work.

\section{References}

[1] Jing Chao, Study on Characteristics of pedestrian crossing traffic, Jilin University Traffic Institute(2007).

[2] Yagil D, Beliefs, in: motives and situational factors related to pedestrians' self-reported behavior at signal-controlled crossings, Transportation Research Part F, Vol. 1-13 (2000)..

[3] Zeeger C V, Stutts J C, et al, in:Analysis of elderly pedestrian accidents and recommended countermeasures, Transportation Research Record, Vol. 56-63 (1993).

[4] Sisiopiku V P, Akin D, in: Pedestrian Behaviors at and Perceptions towards Various Pedestrian Facilities, Transportation Research Part F, Vol. 249-274 (2003).

[5] Hatfield J, and Murphy S, in: The effects of mobile phone use on pedestrian crossing behavior at signalised and unsignalised intersections, Accident Analysis and Prevention, Vol. 197-205 (2007).

[6] Pei Yulong, in: Road Traffic Safety, China Communications press (2004).

[7] Tiwa ri G, Bang diwala S, Sarasw at A, et al, in: Survival analysis: pedestrian risk exposure at signalized inter sections, Transportation Research-F, Vol.77-89 (2007).

[8] Geetam Tiwari, Shfikant Bangdiwala, A ind Saraswat, et.a1, in: Survival analysis-Pedestrian risk exposure at signalized intersections, Transportation Research F, Traffic Psychology and Behaviour, Vol. 77-89 (2007). 